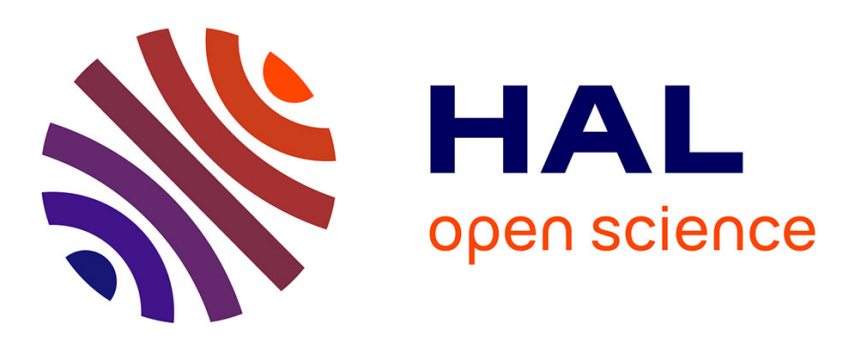

\title{
Dichotomous multi-type games with a coalition structure
} Sébastien Courtin, Zéphirin Nganmeni, Bertrand Tchantcho

\section{To cite this version:}

Sébastien Courtin, Zéphirin Nganmeni, Bertrand Tchantcho. Dichotomous multi-type games with a coalition structure. Mathematical Social Sciences, 2017, 86, pp.9-17. 10.1016/j.mathsocsci.2016.12.003 . halshs-01545772

\section{HAL Id: halshs-01545772 \\ https://shs.hal.science/halshs-01545772}

Submitted on 23 Jun 2017

HAL is a multi-disciplinary open access archive for the deposit and dissemination of scientific research documents, whether they are published or not. The documents may come from teaching and research institutions in France or abroad, or from public or private research centers.
L'archive ouverte pluridisciplinaire HAL, est destinée au dépôt et à la diffusion de documents scientifiques de niveau recherche, publiés ou non, émanant des établissements d'enseignement et de recherche français ou étrangers, des laboratoires publics ou privés. 


\title{
Dichotomous multi-type Games With A CoAlition STRUCTURe
}

\author{
Sébastien Courtin ${ }^{\dagger}$ Zéphirin Nganmeni ${ }^{\ddagger}$ And Bertrand Tchantcho ${ }^{\S}$
}

November 3, 2016

\begin{abstract}
This work focuses on the evaluation of voting power in dichotomous multitype games endowed with a coalition structure. Dichotomous multi-type games, introduced by Courtin et al. [2016], model games in which there is a number of non-ordered types of support in the input, while the output is dichotomous, i.e. the proposal is either accepted or rejected. In a game with a coalition structure, it is supposed that players organize themselves into disjoint coalitions wich are defined a priori. We extend the well-known Owen index (Owen [1977]) and Banzhaf-Owen index (Owen [1981]) to this class of games. A full characterization of these power indices is provided.
\end{abstract}

KEYWORDS: Dichotomous multi-type games, Coalition structure, Owen power index, Banzhaf-Owen power index

JEL Classification Numbers: C71 ;D71.

*The authors would like to thank Fabian Gouret, Annick Laruelle, Mathieu Martin, Matias Nunez, Issofa Moyouwou, three anonymous reviewers and the associate Editor for their useful comments and encouragement. This work has also benefited from comments by a number of conference and seminar participants. This research was carried out within the center of excellence MME-DII (ANR-11-LBX0023-01), and the CoCoRICo-CoDEC research program (ANR-14-CE24-0007-02).

${ }^{\dagger}$ Université de Caen Normandie, CREM UMR CNRS 6211, France. Email: sebastien.courtin@unicaen.fr;

‡Université de Cergy-Pontoise, THEMA, UMR CNRS 8184, France. Email: zephirin.ngameni@ucergy.fr.

§University of Yaounde I, Advanced Teachers' Training College, Cameroon; and Université de Cergy-Pontoise, THEMA, UMR CNRS 8184, France. Email: btchantcho@yahoo.fr; 


\section{Introduction}

How can a voter affect the outcome of an election? This is one of the main concerns in the literature on voting theory, both within political science and in game theory. An analysis of this question can be conducted within the theoretical framework of simple games and power indices. Power indices are quantitative measures of the a priori power of a voter in a committee. Although there are different approaches to how a voter can influence an election ${ }^{1}$ most of the literature concerns a limited scenario. Two main criticisms can be raised to simple games models. The first concerns the number of options offered to players and the second concerns the relationship between players.

Concerning the first criticism, in a simple game, a proposal is approved only on the basis of the votes cast by those who are in favor. In other words, voting "yes" and "no" are the only feasible options. Consequently, the possibility of other voting options such as "abstention" and "non-participation", is not taken into account. It has been pointed out by many scholars that a number of important economic and political games cannot be described by a classical simple game. This is the case for the United Nations Security Council for instance, where "abstention" plays a key role. In response to these limitations, recent years have seen the introduction and analysis of many alternative generalizations of simple games. In this paper, we follow the work of Courtin et al. [2016], who introduced dichotomous multi-type games $(D M G)$. In such games, each player can choose (or not) one of the non-ordered types of support. The players' choices then lead to a choice configuration. The voting rule maps each choice configuration to either a collective approval or a collective disapproval. Consider the following example (Example 1) which is a slight modification of Example 3 in Courtin et al. [2016]. It deals with a committee that must decide on the promotion of a certain junior colleague in an economic department. There are three full professors (namely 1, 2 and 3) and two associate professors (namely 4 and 5). Each professor may not support (NS) or express : a Research Support (RS), a Teaching Support (TS) or an Administrative Support $(A S)$. The candidate is promoted if he receives each of the three types of support, among which at least one $R S$ from a full professor. The associated $D M G$ is such that: (i) the input consists of $N S$ and the three non-ordered types: $R S, T S, A S$ (ii) the output is dichotomous, i.e.

\footnotetext{
${ }^{1}$ See Andjiga et al. [2003] and Laruelle and Valenciano [2008] for a detailed description of the different notions of power indices
} 
the junior colleague is either promoted or not. Clearly, in environment such as the one just described, there is a distinction between the various types of supports and as in this rule, there is no a priori relation between inputs. Particularly, those inputs are not equivalent, which implies that this rule cannot be reduced to a simple game. How can individual power be defined in such environments is therefore an interesting issue. Courtin et al. [2016] extend and fully characterize the Shapley-Shubik (Shapley and Shubik [1954]) and Banzhaf (Banzhaf [1965]) indices for DMG.

In the context of simple games with multiple alternatives, different theories of power have been proposed. The Shapley-Shubik and Banzhaf indices were defined for $(j, k)$ simple games by Freixas [2005a] and Freixas [2005b]. In $(j, k)$ simple games, each player expresses one of $j$ ordered possible levels of input support, and the output consists not of a real value but of one of $k$ possible levels of collective support. $(j, k)$ games is a direct extension of ternary voting games (Felsenthal and Machover [1997]). In a ternary game "abstention" is permitted as a distinct third option for a voter, thus implying three input supports. Clearly, these three input supports are totally ordered in the sense that a "yes" vote is more favorable to the collective acceptance than an "abstention" vote which in turn is more favorable than a "no" vote. Another model of a game with ordered inputs is the multichoice game (Hsiao and Raghavan [1993]). In this game, each player is allowed to have a given number of effort levels, each of which is assigned a nonnegative weight. The weight assigned to an effort level leads to an ordering on the set of effort levels. Any choice configuration is then associated with a real value (in a context of a cooperative game).

Several examples in Freixas and Zwicker [2003] refer to games with inputs not totally ordered with abstention and "non-participation". A formalization of quaternary voting games was provided in Laruelle and Valenciano [2012]. The collective decision is dichotomous, i.e., the proposal is either accepted or rejected. Demanding quorums produces situations without a complete ordering for input levels, see a real-world example in Uleri [2002] and several examples in Freixas and Zwicker [2009]. Indeed, in some situations, the "abstention" option may be more favorable to the rejection of the proposal than the "non-participation" option, while in other situations the converse is observed. However, the "yes" alternative is always more favorable than any other alternative while the "no" alternative is less favorable than any other alternative. This model and the other models above are particular cases of the more general framework of games on lattices developed by Grabisch and Lange [2007]. In this model, each player $i$ has a set of possible actions and this set is en- 
dowed with a lattice, i.e., a partial order such that any pair of actions possesses at least upper bound and a greatest lower bound. Bicooperative games (Bilbao et al. [2000]) can be regarded as a special case of this class of games.

A model of a cooperative game (not only a simple game) which is not based on a lattice is the one of the $r$-games, initially developed by Bolger [1986], Bolger [1993], and later extended by Amer et al. [1998] and Magaña [1996]. In such games, there are $r$ possible input alternatives that are not ordered. Each alternative $j$ attracts its own coalition of supporting voters. A configuration, which is a partition of the set of players into $r$ subsets (some of which might be empty), is then associated with an $r$-tuple of cardinal values. The component $j$ represents the value of the coalition of the configuration that has chosen the input $j$. This model is related to DMG in the sense that the set of inputs is not ordered. No alternative is a priori more favorable than another. However, both models differ in their outputs. Indeed, in a DMG the output consists of a single value.

As far as the a priori relation between different players is concerned, in many negotiation scenarios, players will prefer to cooperate with certain players rather than others. For instance, in the European Union, some States are "pro-European" and others are "opposed to Europe". For example, it is known that France is generally "pro-European", while the UK is more"Euro-skeptic". So when it comes to the scope with questions about the evolution of the EU and the associated institutions, the two countries are more likely to belong to different coalitions. On the contrary, France and Germany are more likely to act together on this subject, since they agree about the evolution of the EU.

One of the sophisticated models which takes these situations into account is that of the game endowed with a priori union. In such a game, there is a partition of the set of players which describes a pre-defined (exogenously given) coalition structure. This strand of the literature was pioneered by Owen [1977] and Owen [1981], who propose and characterize the Owen and the Banzhaf-Owen indices respectively. These two indices are extensions of the Shapley-Shubik and Banzhaf indices respectively to games endowed with a coalition structure.

Our main purpose is to extend and fully characterize the Owen and the BanzhafOwen indices when dichotomous multi-type games are considered. In other words, we extend the notion of $D M G$ to dichotomous multi-type games endowed with a coalition structure (DMGCs).

Note that Albizuri and Zarzuelo [2000] generalize the values considered by Bol- 
ger [1993] when a coalition structure is considered. They consider games with $n$ players and $r$ alternatives, in which the value of a coalition depends not only on that coalition, but also on the organization of the other players in the game. They propose coalitional values that are direct extensions of those of Owen [1977].

This work is structured as follows. Section 2 introduces the general framework. Section 3 presents the main results, while Section 4 concludes the paper. All the proofs are presented in the Appendix.

\section{General framework}

First, we recall the notion of (dichotomous) multi-type games introduced by Courtin et al. [2016]; secondly, we introduce games with coalition structures; and finally we present a different notion of power indices.

\section{(Dichotomous) Multi-type games}

The finite set of players is denoted by $N=\{1,2, \ldots, n\}, \wp(N)$ is the set of all subsets of $N$ and $2^{N}$ is the set of all nonempty subsets of $N: 2^{N}=\wp(N) \backslash\{\varnothing\}$. Any subset $S$ of $N$ is called a coalition. A classical cooperative $n$-person game in characteristic form is a pair $(N, v)$ where $v: \wp(N) \rightarrow \mathbb{R}$ is such that $v(\varnothing)=0$. Such a game is called a simple (voting) game (SG) if : (i) $v(S) \in\{0,1\}$ for all $S \in \wp(N)$; (ii) $v(N)=1$; and (iii) $v$ is monotonic $(v(S) \leq v(T)$ if $S \subseteq T \subseteq N)$. In a $S G$, a coalition $S$ is said to be winning if $v(S)=1$ and losing otherwise.

Let $\bar{R}=\left\{a_{0}, a_{1}, \ldots, a_{k}, \ldots, a_{r}\right\}$, where $r$ is a non-null integer, the set of all possible actions. Note that $a_{0}$ is the option which means no support at all. Each player can then choose between $r+1$ possible actions: no support, or one of the $r$ types of support. For the sake of simplicity and when there is no ambiguity, we write $k \in \bar{R}$ for an element $a_{k} \in \bar{R}$.

A (choice) configuration is a sequence $F=\left(F_{0}, F_{1}, \ldots, F_{r}\right)$, which is a partition of $N$ that is, for all $k \in \bar{R}, F_{k} \subseteq N, \bigcup_{t=1}^{r} F_{t}=N$ and for all $k, j \in \bar{R}, k \neq j \Longrightarrow F_{k} \cap F_{j}=\varnothing . F$ can be seen as a division of the voters according to their action, while $F_{k}$ is the set of voters who choose the action $k \in \bar{R}$. We denote the set of all configurations by $\bar{R}^{N}$.

A multi-type game is a pair $(N, V)$ where $V: \bar{R}^{N} \rightarrow \mathbb{R}$, such that $V(N, \varnothing, \ldots, \varnothing)=0$. Likewise, a dichotomous multi-type game $(D M G)$ is a pair $(N, V)$ such that: (i) $V(F) \in$ $\{0,1\}$ for all $F \in \bar{R}^{N}$; (ii) $V(F)=1$ for at least one $F \in \bar{R}^{N}$; and (iii) $V$ is monotonic (for 
all $\left.F, G \in \bar{R}^{N}, F \subseteq G \Longrightarrow V(F) \leq V(G)\right)^{2}$. A configuration $F$ is a winning if $V(F)=1$ while it is a losing if $V(F)=0$.

We can remark that $F_{0}=N-\bigcup_{k=1}^{r} F_{k}$. This means that $F$ is completely described by $N$ and $\left(F_{k}\right)_{1 \leq k \leq r}$. This allows us to consider in the sequel that a configuration is a sequence $\left(F_{k}\right)_{1 \leq k \leq r}$ and $R=\left\{a_{1}, \ldots, a_{k}, \ldots, a_{r}\right\}$ is the set of types of support, $a_{0}$ being no support at all.The set $N_{F}=\bigcup_{k=1}^{r} F_{k}$ will be referred to as the support of the configuration $F$, which is the set of voters who choose one support action. Clearly a DMG reduces to a $S G$ when $|R|=1$.

\section{(Dichotomous multi-type) Games with coalition structure}

A coalition structure on $N$ is a finite partition $P=\left\{P_{l}: l \in M=\{1, \ldots, m\}\right\}$ of $m$ nonempty and disjoint subsets of $N$, i.e. $\bigcup_{l=1}^{m} P_{l}=N$ and $P_{h} \cap P_{l}=\varnothing$ for all $h, l \in\{1, \ldots, m\}$, $h \neq l$. A coalition structure is assumed to be given exogenously.

A simple game with coalition structure $(S G C s)$, denoted $(v, P)$, is simply a $S G$ which takes into account a given partition of the voters. Let $(v, P)$ be a SGCs and let $l \in M$. The game $(v, P)$ is $l$-anonymous if there exist two positive integers $\mu_{l}$ and $q_{l}$ such that for all $S \subseteq N, v(S)=1$ if and only if

$\left[\left|M\left(S \backslash P_{l}\right)\right| \geq \mu_{l}\right]$ or $\left[\left|M\left(S \backslash P_{l}\right)\right|=\mu_{l}-1\right.$ and $\left.\left|S \cap P_{l}\right| \geq q_{l}\right]$, where for all $T \in 2^{N}, M(T)=$ $\left\{h \in M: P_{h} \subseteq T\right\}$.

In order to understand the concept of $l$-anonymity, let us remark that for such games, a configuration is winning in one of the following conditions, provided that all coalitions in the structure except $P_{l}$ act as a bloc. Either the number of coalitions different from $l$ of the structure who vote for the decision is greater than or equal to a certain threshold $\mu_{l}$ (of course $\mu_{l} \leq m-1$ ) or, this number is exactly $\mu_{l}-1$ and at least $q^{l}$ members of the coalition $P_{l}$ approve the decision. Given a coalition $P_{l}$ in the structure, if the game is $l$-anonymous, then for any configuration $F$, either the number of coalitions in the coalition structure that meets $F^{-l}$ is enough to determine the outcome, or the number of voters of $P_{l}$ who choose each support $k$ is sufficient to determine the outcome.

A dichotomous multi-type game with coalition structure (DMGCs), denoted $(V, P)$, is simply a $D M G$ which takes into account a given partition of the voters.

For all $F \in R^{N}$, we denote by $F^{-l}=\left(F_{k} \backslash P_{l}\right)_{k \in R}$ and we will refer to $M^{r}(F)=\{h \in$ $\left.M: P_{h} \subseteq N_{F}\right\}$ as the set of indices $h \in M$ such that the support for $F, N_{F}$ contains

\footnotetext{
${ }^{2}$ Note that $F \subseteq G$ if for all $k \in R, F_{k} \subseteq G_{k}$.
} 
$P_{h}$. Moreover, in the following, $R^{N \backslash P_{l}}$ will be the set of configurations without $P_{l}$, i.e. such that for all $F \in R^{N}, P_{l} \cap N_{F}=\varnothing$.

Let $(V, P)$ be a DMGCs and let $l \in M .(V, P)$ is said to be $l$-anonymous if there exist integers $\mu_{l} \geq 1$ and $\left(q_{k}^{l}\right)_{k=1, \ldots, r}$ such that for all $F \in R^{N}, V(F)=1$ if and only if $\left[\left|M^{r}\left(F^{-l}\right)\right| \geq \mu_{l}\right]$ or $\left[\left|M^{r}\left(F^{-l}\right)\right|=\mu_{l}-1\right.$ and $\left.\forall k \in R,\left|F_{k} \cap P_{l}\right| \geq q_{k}^{l}\right]$.

The concept of $l$-anonymity for DMGCs is built in the same spirit as for SGCs. In this general case of $r$ types, a configuration is winning if : either the number of coalitions in the structure (different from $l$ ) who vote for the decision is greater than or equal to a certain threshold $\mu_{l}$ or, this number is exactly $\mu_{l}-1$ and at least $q_{k}^{l}$ members of the coalition structure $P_{l}$ choose type $k$. Thus, given a coalition $P_{l}$ in the structure, if the game is $l$-anonymous, then for any configuration $F$, either the number of coalitions in the coalition structure that meet $F^{-l}$ is enough to determine the outcome, or the number of voters of $P_{l}$ who choose each support $k$ is sufficient to determine the outcome.

\section{Power index}

A power index for a $S G C s \varphi$ maps each $S G C S$ to an $n$-dimensional real-valued vector $\varphi(v, P)=\left(\varphi_{1}(v, P), \ldots, \varphi_{n}(v, P)\right)$ such that for all $i, \varphi_{i}(v, P) \in[0,1]$. In this paper, we are mainly concerned with power indices that can be written in the following form. For any game $(v, P)$ and any player $i \in P_{l}$,

$$
\varphi_{i}(v, P)=\sum_{T \in 2_{*}^{N \backslash P_{l}}} \sum_{S \subseteq P_{l}, i \in S} f_{l}(T, S)[v(S \cup T)-v((S-i) \cup T)]
$$

with $2_{*}^{N \backslash P_{l}}=\left\{T \subseteq N \backslash P_{l}: \forall h \in M, P_{h} \cap T \neq \varnothing \Rightarrow P_{h} \subseteq T\right\}$. This is the set of coalitions that (i) do not meet $P_{l}$ and (ii) for any coalition $P_{h}$ of the structure, either contain $P_{h}$ or do not meet $P_{h}$.

It is common to define the worth (power) of a player in terms of his marginal contribution in various coalitions. In $S G C s$, the marginal contribution of any $i \in P_{l}$ is in the form $v(S \cup T)-v((S-i) \cup T)$ with $T \in 2_{*}^{N \backslash P_{l}}, S \subseteq P_{l}$ where it is implicitly assumed that all players in $P_{h}: h \neq l$, act as a bloc. The coefficient $f_{l}(T, S)$ can be seen as a weight that captures the importance of the marginal contribution of player $i$ to $(T, S)$. It depends a priori on $T$ (where $M(T)$ is any subset of $M$ not containing $l$ ) and $S$ and not on $i \in S$ even though the problem is the evaluation of the power of $i$. 
Many power indices for games endowed with coalition structure are constructed in this form. The well-known Owen index (OS), introduced by Owen [1977], which is a generalization of the Shapley-Shubik index is given by:

$$
f_{l}(T, S)=\frac{|M(T)| !(m-|M(T)|-1) !}{m !} \frac{(|S|-1) !\left(\left|P_{l}\right|-|S|\right) !}{\left|P_{l}\right| !} .
$$

Analogously, Owen [1981] presented the Banzhaf-Owen index $(O B)$, an extension of the Banzhaf index to SGCs. In that case,

$$
f_{l}(T, S)=\frac{1}{2^{m-1}} \frac{1}{2^{\left|P_{l}\right|-1}} .
$$

In the following, we call this particular class of power indices the coalition structure weight-dependant power indices (CSWD). In the sequel, we refer to a CSWD power index whenever we consider a power index.

We shall extend the notion of CSWD to DMGCs. But before, we recall the notion of decisiveness that was defined in Courtin et al. [2016]. Given a $D M G$ and a configuration $F$, a player $i$ is said to be decisive in $F$ if $[V(F)-\bar{V}(F-i)]=1$ with $\bar{V}(F)=\underset{Z \in R^{N}}{\operatorname{Max}}\left\{V(Z), N_{Z}=N_{F}\right\}$ and $F-i=\left(F_{k}-i\right)_{k \in R}$.

In Example 1 developed in the introduction, the candidate is promoted if he receives each of the three types of support, among which at least one $R S$ from a full professor. Consider the following two configurations:

\begin{tabular}{|l|l|l|l|l|}
\hline & $N S$ & $R S$ & $T S$ & $A S$ \\
\hline$F$ & $\{1\}$ & $\{2\}$ & $\{3,4\}$ & $\{5\}$ \\
\hline
\end{tabular}

\begin{tabular}{|l|l|l|l|l|}
\hline & $N S$ & $R S$ & $T S$ & $A S$ \\
\hline$G$ & $\{1,2\}$ & $\{3\}$ & $\{4\}$ & $\{5\}$ \\
\hline
\end{tabular}

It is clear that for both configurations, the candidate is promoted.

Player 2 (for example) is not decisive in $F$ because even though $\bar{V}(F-2)=0$, we have $\bar{V}(F-2)=1$. On the other hand, players 3,4 and 5 are decisive in $G$.

In the context of $D M G C s$, we propose the following form for a CSWD power index for a player $i \in P_{l}, l \in M$ :

$$
\Phi_{i}(V, P)=\sum_{L \in R_{*}^{N \backslash P_{l}}}\left(\sum_{G \in R^{P_{l}}} f_{l}(L, G)[V(L \cup G)-\bar{V}(L \cup(G-i))]\right)
$$

with $R_{*}^{N \backslash P_{l}}=\left\{F \in R^{N \backslash P_{l}}: \forall h \in M, l \neq h, P_{h} \cap N_{F} \neq \varnothing \Longrightarrow P_{h} \subseteq N_{F}\right\}$.

The set $R_{*}^{N \backslash P_{l}}$ contains all configurations $F$ such that (i) the support does not meet 
$P_{l}$ and (ii) for any coalition $P_{h}$ of the structure, the support either contains $P_{h}$ or does not meet $P_{h}$.

Note that for $|R|=1, \Phi_{i}(V, P)=\varphi_{i}(v, P)$ for a player $i$.

\section{Power index for DMGCs}

In this section, we first outline an axiomatic approach to the Owen power index both for SGCs and DMGCs. Following the same approach, we then present an alternative characterization of the Banzhaf-Owen power index.

\section{Owen index for $S G C s$}

Axiom 1. Equal Share for l-anonymous SGCs

A power index $\varphi$ satisfies the Equal Share axiom if for all l-anonymous SGCs, for all $l \in M$, and for all $i \in P_{l}$,

$$
\varphi_{i}(v, P)=\frac{1}{m\left|P_{l}\right|} .
$$

Equal share is a sort of amalgamation of the classical efficiency and symmetry conditions. The Equal Share axiom for SGCs means that in an $l$-anonymous $S G C s$, in any coalition $P_{l}$, voters have the same power. Moreover, this power is proportional to the size of the coalition structure and to the number of voters in $P_{l}$. Note that Equal Share does not mean that voters in $N$ have the same power. Instead, all coalitions

in the structure have the same power $\frac{1}{m}$, which is equally allocated to voters in the same coalition.

Axiom 2. Equal Size for SGCs

A power index $\varphi$ satisfies the Equal Size axiom if for all $l \in M$, for all $T_{1}, T_{2} \in 2_{*}^{N \backslash P_{l}}$ and for all $S_{1}, S_{2} \subseteq P_{l}$,

$$
\left[\left|M\left(T_{1}\right)\right|=\left|M\left(T_{2}\right)\right| \text { and }\left|S_{1}\right|=\left|S_{2}\right|\right] \Longrightarrow f_{l}\left(T_{1}, S_{1}\right)=f_{l}\left(T_{2}, S_{2}\right)
$$

Let $(T, S)$ such that $T \in 2_{*}^{N \backslash P_{l}}$ and $S \subseteq P_{l}$ : recall that with respect to $T$, coalitions of the partition behave as blocs. In other words either $T$ does not meet $P_{h}$ or $T$ contains $P_{h}$ as a whole. Equal Size means that the sizes and identities of members of different coalitions in the partition do not matter. The weight of $(T, S)$ depends (solely) on $l$ (the coalition of the structure that contains $S$ ), the number of indices $k \in M$ such that $P_{k} \subseteq T$ (that is $|M(T)|$ ) and the size of the subset $S$ of $P_{l}$. 
We can now characterize $O S$ in the class of CSWD power indices.

Theorem 1. The Owen power index is the only CSWD power index satisfying the Equal Share and Equal Size axioms.

The theorem above presents an alternative characterization of OS, which is based on the characterization of Shapley-Shubik given by Courtin et al. [2016].

\section{Owen index for DMGCs}

The extension of the Equal Share axiom to DMGCs is straightforward. Once again, in an $l$-anonymous DMGCs, the power of each voter in $P_{l}$ is the same and is proportional to both the number of a priori coalitions and to the number of voters in $P_{l}$.

Axiom 3. Equal Share for DMGCs

A power index $\Phi$ satisfies the Equal Share axiom if for all l-anonymous DMGCs, for all $l \in M$, and for all $i \in P_{l}$,

$$
\Phi_{i}(V, P)=\frac{1}{m\left|P_{l}\right|} .
$$

Likewise, we extend below the Equal Size axiom to DMGCs.

Axiom 4. Equal Size for DMGCs

A power index $\Phi$ satisfies the Equal Size axiom if for all $l \in M$, for all $L^{1}, L^{2} \in R_{*}^{N \backslash P_{l}}$, and for all $G^{1}, G^{2} \in R^{P_{l}}$,

$\left[\left|M^{r}\left(L^{1}\right)\right|=\left|M^{r}\left(L^{2}\right)\right|\right.$ and $\left.\forall k \in R,\left|G_{k}^{1}\right|=\left|G_{k}^{2}\right|\right] \Longrightarrow f_{l}\left(L^{1}, G^{1}\right)=f_{l}\left(L^{2}, G^{2}\right)$.

According to the Equal Size axiom, two couples $\left(L^{1}, G^{1}\right)$ and $\left(L^{2}, G^{2}\right)$ that contain the same number of elements must be equally weighted. This axiom has a very similar interpretation to that presented in the context of SGCs. The only difference is that, instead of considering couples $(T, S)$ such that $T \in 2_{*}^{N \backslash P_{l}}$ and $S \subseteq P_{l}$, we consider couples $(L, G)$ where $L \in R_{*}^{N \backslash P_{l}}$ and $G \in R^{P_{l}}, G$ being a configuration of $P_{l}$.

Before going on to present a generalization of Theorem 1 , let us denote by $[L]_{l}$, for a given $l \in M$, the set of family of indices $I$ without $l$, for which both the cardinalities of $M^{r}(L)$ and $I$ coincide, formally, $[L]_{l}=\left\{I \subseteq M \backslash l:\left|M^{r}(L)\right|=|I|\right\}$.

Theorem 2. There exists one and only one CSWD power index for DMGCs satisfying the Equal Share and the Equal Size axioms. 
This CSWD is such that: for all DMGCs $(V, P)$, all $l \in M$, all $L \in R_{*}^{N \backslash P_{l}}$ and all $G \in R^{P_{l}}$,

$$
f_{l}(L, G)=\left(m \sum_{I \in[L]_{l}} r^{\left(\sum_{h \in I}\left|P_{h}\right|\right)}\right)^{-1}\left(\frac{\left(\left|P_{l}\right|-\sum_{k \in R}\left|G_{k}\right|\right) !\left(\prod_{k \in R}\left|G_{k}\right| !\right)}{\left|P_{l}\right| !\left(\sum_{k \in R}\left|G_{k}\right|\right)}\right)
$$

We can now define the Owen power index for DMGCs. Let $(V, P)$ be a multi-type game endowed with a coalition structure $P=\left\{P_{1}, \ldots, P_{m}\right\}$ and let $i \in N$. The Owen power index of player $i$ in $(V, P)$ is given by:

$$
\begin{aligned}
& O S_{i}^{r}(V, P)= \\
& \sum_{L \in R_{*}^{N \backslash P_{l}}}\left(\sum_{G \in R^{P_{l}}}\left(m \sum_{I \in[L]_{l}} r_{h \in I}^{\left(\sum_{h \in I}\left|P_{h}\right|\right)}\right)^{-1}\left(\frac{\left(\left|P_{l}\right|-\sum_{k \in R}\left|G_{k}\right|\right) !\left(\prod_{k \in R}\left|G_{k}\right| !\right)}{\left|P_{l}\right| !\left(\sum_{k \in R}\left|G_{k}\right|\right)}\right)\right) \times(V(L \cup G)-\bar{V}(L \cup(G-i)))
\end{aligned}
$$

Corollary 1. If $|R|=1$, then the Owen power index for DMGCs is equivalent to the Owen power index for SGCs.

\section{Banzhaf-Owen power index for SGCs and DMGCS}

We now provide a characterization of the class of CSWD power indices based on the famous Banzhaf score (Banzhaf [1965]). Given a $S G$, the Banzhaf score of a player $i$ in this game is the number of coalitions in which he is decisive $\left(\sum_{S \in 2^{N}}[v(S)-v(S-i)]\right)$. Hence, for all $i \in P_{l}$, the generalization to a $S G C S$ of the Banzhaf score of a player $i$, is the number of $(T, S) \in 2_{*}^{N \backslash P_{l}} \times P_{l}$ such that $[v(S \cup T)-v((S-i) \cup T)]=1$. More formally, for all $i \in P_{l}$,

$$
\eta_{i}(v, P)=\sum_{T \in 2_{*}^{N} \backslash P_{l} S \subseteq P_{l}, i \in S}[v(S \cup T)-v((S-i) \cup T)]
$$

Axiom 5. Constant-weight axiom for SGCs

A power index $\varphi$ satisfies the Constant Weight axiom if for all $l \in M$, there exists $\lambda^{l}>0$ such that for all $T \in 2_{*}^{N \backslash P_{l}}$ and for all $S \subseteq P_{l}$,

$$
f_{l}(T, S)=\lambda^{l}
$$


Note that if this is the case then the power index is said to be a $\left(\lambda^{l}\right)$-constantweight.

The weight associated to each couple $(T, S), T \in 2_{*}^{N \backslash P_{l}}$ and $S \subseteq P_{l}$ is always the same. This means that for power index satisfying Constant-weight axiom, the weight of $(T, S)$ depends solely on the coalition $P_{l}$ which includes $S$.

We obtain the following proposition.

Proposition 1. A power index for SGCs is $\left(\lambda^{l}\right)$-constant-weight if and only if for all $(v, P)$ and for all $i \in P_{l}$,

$$
\psi_{i}(v, P)=\lambda^{l} \eta_{i}(v, P)
$$

Let us remark that for $\lambda^{l}=\frac{1}{2^{m-1} \times 2^{\left|P_{l}\right|-1}}$, we obtain a new characterization of BanzhafOwen.

We can also extend the Banzhaf scores to DMGCs. Let $(V, P)$ be a DMGCs, $i \in P_{l}$, the Banzhaf score of $i$ is given by

$$
\eta_{i}^{r}(V, P)=\sum_{L \in R_{*}^{N \backslash P_{l}}} \sum_{G \in R^{P_{l}}}[V(L \cup G)-\bar{V}(L \cup(G-i))] .
$$

\section{Axiom 6. Constant-weight axiom for DMGCs}

A power index $\Psi$ for DMGCs satisfies the Constant Weight axiom if for all $l \in M$, there exists $\delta^{l}>0$ such that for all $L \in R_{*}^{N \backslash P_{l}}$ and for all $G \in R^{P_{l}}, f_{l}(L, G)=\delta^{l}$.

Once more, the Constant-weight axiom defined for SGCs is extended to DMGCs by merely replacing $(T, S)$ (where $T \in 2_{*}^{N \backslash P_{l}}$ and $S \subseteq P_{l}$ ) with $(L, G)$ where $L \in R_{*}^{N \backslash P_{l}}$ and $G \in R^{P_{l}}$. The following theorem is a direct extension of Proposition 1.

Proposition 2. A CSWD power index for DMGCs is $\left(\delta^{l}\right)$-constant-weight if and only if for all DMGCs $(V, P)$, and for all $i \in P_{l}, \Phi_{i}(V, P)=\delta^{l} \eta_{i}^{r}(V, P)$.

Consequently, for $\delta^{l}=\frac{1}{(r+1)^{m-1} \times(r+1)^{\left|P_{l}\right|-1}}$, we obtain a generalization of $O B$. More formally, for a player $i \in P_{l}$, we have

$$
O B_{i}^{r}(V, P)=\frac{\sum_{L \in R_{*}^{N \backslash P_{l}}} \sum_{G \in R^{P_{l}}}[V(L \cup G)-\bar{V}(L \cup(G-i))]}{(r+1)^{m-1} \times(r+1)^{\left|P_{l}\right|-1}}
$$

To conclude, let us compute the Owen and the Banzhaf-Owen indices in Example 1. For this, consider that among the three full Professors, two of them organize 
themselves to defend their interests. Likewise, the two associate Professors form an a priori coalition. The last full Professor 3 stays alone, leading to $P=\{(1,2),(3),(4,5)\}$.

i) Let us consider the $S G C s$ in which the candidate is promoted if at least three professors vote "for", among which at least one full professor. This means that we assume that all the (three) types of support are "equivalent".

In this case, we obtain $O S(v, P)=\left(\frac{1}{6}, \frac{1}{6}, \frac{2}{6}, \frac{1}{6}, \frac{1}{6}\right)$ and $O B(v, P)=\left(\frac{1}{4}, \frac{1}{4}, \frac{2}{4}, \frac{1}{4}, \frac{1}{4}\right)$.

ii) Now let us come back to the game considering the three type of support. The computation of the power of each professor yields the following:

$O S^{r}(V, P)=\left(\frac{15}{216}, \frac{15}{216}, \frac{32}{216}, \frac{15}{216}, \frac{15}{216}\right)$ while $O B^{r}(V, P)=\left(\frac{4}{32}, \frac{4}{32}, \frac{16}{32}, \frac{3}{32}, \frac{3}{32}\right)$.

These results show that the computation of $O S^{r}(V, P)$ and $O B^{r}(V, P)$ captures the different types of input support. Indeed, in the class of CSWD power index, the power of a player is the sum of the weights of all configurations where he is decisive. In a multi-type game, one can assess the fraction of the sum associated with each type. The numbers obtained above are explained by the following table :

\begin{tabular}{|c|c|c|c|}
\hline$O S_{i}^{r}(V, P)$ & $R S$ & $T S$ & $A S$ \\
\hline $1 ; 2$ & $\frac{9}{216}$ & $\frac{3}{216}$ & $\frac{3}{216}$ \\
\hline 3 & $\frac{16}{216}$ & $\frac{8}{216}$ & $\frac{8}{216}$ \\
\hline $4 ; 5$ & 0 & $\frac{7.5}{216}$ & $\frac{7.5}{216}$ \\
\hline
\end{tabular}

\begin{tabular}{|c|c|c|c|}
\hline$O B_{i}^{r}(V, P)$ & $R S$ & $T S$ & $A S$ \\
\hline $1 ; 2$ & $\frac{2}{32}$ & $\frac{1}{32}$ & $\frac{1}{32}$ \\
\hline 3 & $\frac{8}{32}$ & $\frac{4}{32}$ & $\frac{4}{32}$ \\
\hline $4 ; 5$ & 0 & $\frac{1.5}{32}$ & $\frac{1.5}{32}$ \\
\hline
\end{tabular}

For example, with respect to $\operatorname{OS}^{r}(V, P), \frac{9}{216}$ represents the sum of weights of configurations in which player 1 (or 2 ) is decisive with the type $R S$. As well, $\frac{3}{216}$ represents the sum of weights of configurations in which player 1 (or 2) is decisive with the type TS. Other rows can be interpreted as well. One can observe that players 1, 2 and 3 obtain larger fraction from $R S$. This is certainly explained by the voting rule.

\section{Conclusion}

This work deals with dichotomous multi-type support games in the context of a priori coalitions between players. It is worth noting that simple games with coalition structure do not allow for voters to express different types of support. The extension of the classical notions of coalition structure voting power to such games was the main objective of this paper. Thanks to alternative characterizations of the Owen and Banzhaf-Owen power indices, we provide full characterizations of these indices 
in our framework. Note that when considering the trivial partition, $P=\left\{\{i\}_{i \in N}\right\}$, Owen and Banzhaf-Owen indices for DMGCs reduce respectively to the Shapley and Banzhaf indices for DMG, introduced by Courtin et al. [2016].

There are several ways in which dichotomous multi-type games could be explored in further research. Firstly, we can extend the notion of the desirability relation, to qualitatively compare the a priori influence of voters in DMGCs. The desirability relation was extended in games with abstention by Tchantcho et al. [2008]. In the context of SGCs, Courtin and Tchantcho [2015] already shows that $O S$ and $O B$ are not ordinally equivalent. Secondly, following the works of Albizuri et al. [2006] and Albizuri and Aurrekoetxea [2006], we can also provide power indices in the context of dichotomous multi-type games with coalition configurations. In games with coalition configurations, the players organize themselves into coalitions that are not necessarily disjoint. Finally, in our framework we can conduct an analysis of the concept of manipulation, as studied by Freixas and Parker [2015] in the context of $(j, k)$ games.

\section{References}

M.J. Albizuri and J. Aurrekoetxea. Coalition configurations and the Banzhaf index. Social Choice and Welfare, 26:571-596, 2006.

M.J. Albizuri and J.M. Zarzuelo. Coalitional values for cooperative games with $\mathrm{r}$ alternatives. TOP, 8:1-30, 2000.

M.J. Albizuri, J. Aurrekoetxea, and J.M. Zarzuelo. Configuration values: extensions of the coalitional Owen value. Games and Economic Behavior, 57:1-17, 2006.

R. Amer, F. Carreras, and A. Magaña. Extension of values to games with multiple alternatives. Annals of Operations Research, 84:63-78, 1998.

N. Andjiga, F. Chantreuil, and D. Lepelley. La mesure du pouvoir de vote. Mathematiques et Sciences Humaines, 163:111-145, 2003.

J.F. Banzhaf. Weighted voting doesnt work: A mathematical analysis. Rutgers Law Review, 19:317-343, 1965. 
J.M. Bilbao, J.R. Fernández, A. Jiménez Losada, and E. Lebron. Bicooperative games. In J.M. Bilboa, editor, Cooperative Games on Combinatorial Structures. Kluwer, Dordrecht, 2000.

E.M. Bolger. Power indices for multicandidate voting games. International Journal of Game Theory, 15:175-186, 1986.

E.M. Bolger. A value for games with $\mathrm{n}$ players and $\mathrm{r}$ alternatives. International Journal of Game Theory, 22:319-334, 1993.

S. Courtin and B. Tchantcho. A note on the ordinal equivalence of power indices in games with coalition structure. Theory and Decision, 78:617-628, 2015.

S. Courtin, Z. Nganmeni, and B. Tchantcho. The Shapley-Shubik power index for dichotomous multi-type games:. Theory and Decision, 10.1007/s11238-016-95414, 2016.

D.S. Felsenthal and M. Machover. Ternary voting games. International Journal of Game Theory, 26:335-351, 1997.

J. Freixas. The Banzhaf index for games with several levels of approval in the input and output. Annals of Operations Research, 137:45-66, 2005a.

J. Freixas. The Shapley Shubik power index for games with several levels of approval in the input and output. Decision Support Systems, 39:185-195, 2005b.

J. Freixas and C. Parker. Manipulation in games with multiple levels of output. Journal of Mathematical Economics, 61:144-151, 2015.

J. Freixas and W. S. Zwicker. Weighted voting, abstention, and multiple levels of approval. Social Choice and Welfare, 21:399-431, 2003.

J. Freixas and W. S. Zwicker. Anonymous yes-no voting with abstention and multiple levels of approval. Games and Economic Behavior, 67(2):428-444, 2009.

M. Grabisch and F. Lange. Games on lattices, multichoice games and the Shapley value: a new approach. Mathematical Methods of Operations Research, 65:153-167, 2007.

C.R. Hsiao and T.E.S. Raghavan. Shapley value for multichoice cooperative games 1. Games and Economic Behavior, 5:240-256, 1993. 
A. Laruelle and F. Valenciano. Voting and collective decision-making. Cambridge University Press, 1st edition, 2008.

A. Laruelle and F. Valenciano. Quaternary dichotomous voting rules. Social Choice and Welfare, 38:431-454, 2012.

A. Magaña. Formacion de coaliciones en los juegos cooperativos y juegos con multiples alternativas. PhD Thesis, Mathematics Department of UPC, Spain, 1996.

G. Owen. Values of games with a priori unions. In R. Hein and O. Moeschlin, editors, Essays in Mathematical Economics and Game Theory. Springer, New York, 1977.

G. Owen. Modification of the Banzhaf-Coleman index for games with a priori unions. In M.J. Holler, editor, Power, Voting, and Voting Power. Physica-Verlag, Wurzburg, 1981.

L. S. Shapley and M. Shubik. A model for evaluating the distribution of power in a committee system. Rutgers Law Review, 48:787-792, 1954.

B. Tchantcho, L. Diffo Lambo, R. Pongou, and B. Mbama Engoulou. Voters' power in voting games with abstention: Influence relation and ordinal equivalence of power theories. Games and Economic Behavior, 64:335-350, 2008.

P. V. Uleri. On referendum voting in italy: Yes, no or non-vote? how italian parties learned to control referendums. European Journal of Political Research, 41(6):863883,2002 . ISSN 1475-6765.

\section{A Appendix}

\section{A.1 Proof of Theorem 1}

The following straightforward lemma will be useful in order to prove the theorem.

Lemma 1. Let $(v, P)$ be a $S G C s, l \in M, T \in 2_{*}^{N \backslash P_{l}}$ and $S \subseteq P_{l}$.

If $(v, P)$ is l-anonymous with coefficient factors $\mu_{l}$ and $q_{l}$, then for all $i \in P_{l}$, $v(S \cup T)-v((S-i) \cup T)=1 \Longleftrightarrow i \in S,|S|=q_{l}$ and $|M(T)|=\mu_{l}-1$. 


\section{Proof. Theorem 1}

$\Longrightarrow$ ) Let $\varphi$ be a CSWD power index that satisfies the Equal Share and Equal Size axioms. We shall show that $\varphi$ is the Owen power index. It is equivalent to show that for all $l \in M$, all $T \in 2_{*}^{N \backslash P_{l}}$ and all $S \subseteq P_{l}$, the factor $f_{l}(T, S)$ associated with $\varphi$ is given by: $f_{l}(T, S)=\frac{\mid M(T) !(m-|M(T)|-1) !}{m !} \frac{(|S|-1) !\left(\left|P_{l}\right|-|S|\right) !}{\left|P_{l}\right| !}$. Let $l \in M, T \in 2_{*}^{N \backslash P_{l}}$ and $S \subseteq P_{l}$, consider the $S G C s(v, P)$ defined by: for all $\Lambda \subseteq N, v(\Lambda)=1$ if and only if $\left|M\left(\Lambda \backslash P_{l}\right)\right| \geq|M(T)|+1$ or $\left[\left|M\left(\Lambda \backslash P_{l}\right)\right|=|M(T)|\right.$ and $\left.\left|\Lambda \cap P_{l}\right| \geq|S|\right]$. It follows that $(v, P)$ is $l$-anonymous with the coefficients factors $|M(T)|+1$ and $|S|$. Let $i \in P_{l}$, set $W_{i}^{l}=\left\{(Z, \Lambda): Z \in 2_{*}^{N \backslash P_{l}}, \Lambda \subseteq P_{l}, v(\Lambda \cup Z)-v((\Lambda-i) \cup Z)=1\right\}$.

By lemma $1, W_{i}^{l}=\left\{(Z, \Lambda): Z \in 2_{*}^{N \backslash P_{l}}, \Lambda \subseteq P_{l}, i \in \Lambda,|M(Z)|=|M(T)|\right.$ and $\left.|\Lambda|=|S|\right\}$, which leads to $\left\{Z \in 2_{*}^{N \backslash P_{l}},|M(Z)|=|M(T)|\right\} \times\left\{\Lambda \subseteq P_{l}, i \in T,|\Lambda|=|S|\right\}$. Moreover,

$$
\varphi_{i}(v, P)=\sum_{Z \in 2_{*}^{N \backslash P_{l}} \Lambda \subseteq P_{l}, i \in \Lambda} f_{l}(Z, \Lambda)[v(\Lambda \cup Z)-v((\Lambda-i) \cup Z)]=\sum_{(Z, \Lambda) \in W_{i}^{l}} f_{l}(T, S) \text {. Since }
$$

for all $(Z, \Lambda) \in W_{i}^{l}$, we have $|M(Z)|=|M(T)|$ and $|\Lambda|=|S|$. By Equal Size, it holds $f_{l}(Z, \Lambda)=f_{l}(T, S)$; thus, $\varphi_{i}(v, P)=\sum_{(Z, \Lambda) \in W_{i}^{l}} f_{l}(T, S)=f_{l}(T, S)\left|W_{i}^{l}\right|$.

But $\left|W_{i}^{l}\right|=\left|\left\{Z \in 2_{*}^{N \backslash P_{l}},|M(Z)|=|M(T)|\right\}\right| \times\left|\left\{\Lambda \subseteq P_{l}, i \in T,|\Lambda|=|S|\right\}\right|=C_{m-1}^{|M(T)|} C_{\left|P_{l}\right|}^{|S|-1}$. Thus, $\varphi_{i}(v, P)=f_{l}(T, S) \frac{(m-1) !}{\mid M(T) ! !(m-|M(T)|-1) !} \frac{\left|P_{l}-1\right| !}{(|S|-1) !\left(\left|P_{l}\right|-|S|\right) !}$. By Equal Share, $\forall i \in P_{l}$, $\varphi_{i}(v, P)=\frac{1}{m\left|P_{\mid}\right|}$, it follows that $f_{l}(T, S) \frac{(m-1) !}{\mid M(T) !(m-|M(T)|-1) !} \frac{\left|P_{l}-1\right| !}{(|S|-1) !\left(\left|P_{l}\right|-|S|\right) !}=\frac{1}{m\left|P_{l}\right|}$, which implies that $f_{l}(T, S)=\frac{\mid M(T) !(m-|M(T)|-1) !}{m !} \frac{(|S|-1) !\left(\left|P_{l}\right|-|S|\right) !}{\left|P_{l}\right| !}$.

$\Leftrightarrow)$ Conversely, the CSWD power index for which the factor of a given $(T, S)$ is $f_{l}(T, S)=\frac{\mid M(T) !(m-|M(T)|-1) !}{m !} \frac{(|S|-1) !\left(\left|P_{l}\right|-|S|\right) !}{\left|P_{l}\right| !}$ is clearly the Owen power index, which obviously satisfies the Equal Share and Equal Size axioms.

\section{A.2 Proof of Theorem 2}

We need two lemmas in order to prove this theorem. The first one is taken from Courtin et al. [2016] and is recalled below.

Lemma 2. Courtin et al. [2016]

Let $N$ be a set of $n$ players and $\left(n_{t}\right)$ a sequence of $r$ integers such that $\sum_{t=1}^{r} n_{t}=n$. The number of partitions of $N$ into $r$ coalitions $S_{1}, \ldots, S_{r}$ such that for all $t=1,2, \ldots, r,\left|S_{t}\right|=n_{t}$, is given by the formula $\frac{n !}{n_{1} ! \times n_{2} ! \times \ldots \times n_{n} !}$.

Lemma 3. Let $(V, P)$ be a DMGCs, $l \in M,, F \in R_{*}^{N \backslash P_{l}}$ and $G \in R^{P_{l}}$. 
If $(V, P)$ is l-anonymous, with the coefficients $\mu_{l},\left(q_{k}^{l}\right)_{k \in R}$ then: for all $i \in N_{G}$ $V(F \cup G)-\bar{V}(F \cup(G-i))=1 \Longleftrightarrow\left|M^{r}(F)\right|=\mu_{l}-1$ and $\forall k \in R,\left|G_{k}\right|=q_{k}^{l}$.

Proof. Lemma 3

Let $(V, P)$ be a DMGCs, $l \in M, i \in P_{l}, F \in R_{*}^{N \backslash P_{l}}$ and $G \in R^{P_{l}}$. Assume that $(V, P)$ is $l$-anonymous, with the coefficients factors $\mu_{l},\left(q_{k}^{l}\right)_{k \in R}$

$\Rightarrow$ ) Assume that $V(F \cup G)-\bar{V}(F \cup(G-i))=1$, we shall show that $\left|M^{r}(F)\right|=\mu_{l}-1$ and $\forall k \in R,\left|G_{k}\right|=q_{k}^{l}$. We know that $V(F \cup G)-\bar{V}(F \cup(G-i))=1$ if and only if $V(F \cup G)=1$ and $\bar{V}(F \cup(G-i))=0$ (1). Moreover, $\bar{V}(F \cup(G-i))=0$ means that for all $H \in R^{N}$, if $N_{H}=N_{(F \cup(G-i))}$ then $V(H)=0(2)$. By hypothesis, $(V, P)$ is $l$ anonymous, with the coefficients $\mu_{l},\left(q_{k}^{l}\right)_{k \in R^{\prime}}$, thus:

$$
\begin{aligned}
& V(F \cup G)=1 \Longrightarrow\left\{\begin{array}{l}
\left|M^{r}\left((F \cup G)^{-l}\right)\right| \geq \mu_{l} \text { or } \\
\left.|| M^{r}\left((F \cup G)^{-l}\right) \mid=\mu_{l}-1 \text { and } \forall k \in R,\left|\left(F_{k} \cup G_{k}\right) \cap P_{l}\right| \geq q_{k}^{l}\right]
\end{array}\right. \\
& \Longrightarrow\left\{\begin{array}{l}
\left|M^{r}(F)\right| \geq \mu_{l} \text { or } \\
{\left[\left|M^{r}(F)\right|=\mu_{l}-1 \text { and } \forall k \in R,\left|G_{k}\right| \geq q_{k}^{l}\right]}
\end{array}\right.
\end{aligned}
$$

We claim that $\left|M^{r}(F)\right| \geq \mu_{l}$ is impossible. Indeed, since $F^{-l}=F,\left|M^{r}(F)\right| \geq \mu_{l}$ implies that $\left|M^{r}\left(F^{-l}\right)\right| \geq \mu_{l}$ and that $V(F)=1$ by $(V, P) l$-anonymous. Furthermore, $F \subseteq F \cup(G-i)$; therefore $V(F \cup(G-i))=1$ and hence $\bar{V}(F \cup(G-i))=1$. This is a contradiction with (1). It then follows from (3) that $\left[\left|M^{r}(F)\right|=\mu_{l}-1\right.$ and $\left.\forall k \in R,\left|G_{k}\right| \geq q_{k}^{l}\right]$. We prove now that $\forall k \in R,\left|G_{k}\right|=q_{k}^{l}$. Assume on the contrary that there exists $e \in R$ such that $\left|G_{e}\right|>q_{e}^{l}$. Consider the integer $j \in R$ such that $i \in G_{j}$ :

Let $i^{\prime} \in G_{e}$ and $L \in R^{P_{l}}$ defined by $\forall k \in R, L_{k}=\left\{\begin{array}{l}G_{k} \backslash\left\{i, i^{\prime}\right\} \text { if } k \neq j \\ \left(F_{k} \backslash\{i\}\right) \cup\left\{i^{\prime}\right\} \text { if } k=j\end{array}\right.$

It is easy to show that: $\forall k \in R,\left|L_{k}\right| \geq q_{k}^{l}$. It follows that $V(F \cup L)=1$. On the other hand, $N_{F \cup L}=N_{(F \cup(G-i))}$ and thanks to (2) it follows that $V(F \cup L)=0$, which is a contradiction. We deduce that $\left|M^{r}(F)\right|=\mu_{l}-1$ and $\forall k \in R,\left|G_{k}\right|=q_{k}^{l}$, which concludes the first part of the proof.

$\Leftarrow)$ Assume that $\left|M^{r}(F)\right|=\mu_{l}-1$ and $\forall k \in R,\left|G_{k}\right|=q_{k}^{l}$. Let us prove that $V(F \cup$ $G)-\bar{V}(F \cup(G-i))=1$. We have $\left|M^{r}(F)\right|=\mu_{l}-1$ and $\forall k \in R,\left|G_{k}\right|=q_{k}^{l}$ thus, by $(V, P) l-$ anonymous, it follows that $V(F \cup G)=1$. Let $H \in R^{N}$ such that $N_{H}=N_{(F \cup(G-i))}$. Then, $\left|M^{r}\left(H^{-l}\right)\right|=\left|M^{r}\left((F \cup(G-i))^{-l}\right)\right|=\left|M^{r}(F)\right|=\mu_{l}-1$. Furthermore, $\underset{k \in R}{\cup}\left(H_{k} \cap P_{l}\right)=G-i$ since $N_{H}=N_{(F \cup(G-i))}$. Hence, $\sum_{k \in R}\left|H_{k} \cap P_{l}\right|=|(G-i)|<|G|=\sum_{k \in R}\left|G_{k}\right|=\sum_{k \in R} q_{k}^{l}$. Therefore, 
$\sum_{k \in R}\left(\left|H_{k} \cap P_{l}\right|-q_{k}^{l}\right)<0$, which means that there exists $j \in R:\left|H_{j} \cap P_{l}\right|<q_{j}^{l}$.

To summarize, we have: $\left|M^{r}(F)\right|=\mu_{l}-1,\left|H_{j} \cap P_{l}\right|<q_{j}^{l}$ and $(V, P)$ is $l$-anonymous with coefficients $\mu_{l},\left(q_{k}^{l}\right)_{k \in R}$. It follows that $V(H)=0$ for all $H \in R^{N}$ such that $N_{H}=$ $N_{(F \cup(G-i))}$, consequently $\bar{V}(F \cup(G-i))=0$ and we get $V(F \cup G)-\bar{V}(F \cup(G-i))=1$.

We can now prove Theorem 2.

Proof. Theorem 2

$\Rightarrow$ Let $\Phi$ be a CSWD power index which verifies the Equal Share and Equal Size properties, with $\left(f_{l}(L, G)\right)_{(L, G)}$. Let $l \in M, L \in R^{N \backslash P_{l}}$ and $G \in R^{P_{l}}$; we will show that:

$$
f_{l}(L, G)=\left(m \sum_{I \in[L]_{l}} r\left(\sum_{h \in I}\left|P_{h}\right|\right)\right)^{-1}\left(\frac{\left(\left|P_{l}\right|-\sum_{k \in R}\left|G_{k}\right|\right) !\left(\prod_{k \in R}\left|G_{k}\right| !\right)}{\left|P_{l}\right| !\left(\sum_{k \in R}\left|G_{k}\right|\right)}\right)
$$

Define $(V, P)$ such that: $\forall F \in R^{N}, V(F)=1$ if and only if $\left|M^{r}\left(F^{-l}\right)\right| \geq\left|M^{r}(L)\right|+1$ or $\left[\left|M^{r}\left(F^{-l}\right)\right|=\left|M^{r}(L)\right|\right.$ and $\left.\forall k \in R,\left|F_{k} \cap P_{l}\right| \geq\left|G_{k}\right|\right]$. It follows that $(V, P)$ is $l$-anonymous with the coefficients $\left|M^{r}(L)\right|+1,\left(\left|G_{k}\right|\right)_{k \in R}$. Let $i \in N$ and set:

$$
\begin{aligned}
\bar{W}_{l}^{i} & =\left\{(F, H): F \in R_{*}^{N \backslash P_{l}}, H \in R^{P_{l}} \text { and } V(F \cup H)-\bar{V}(F \cup(H-i))=1\right\} \\
& =\left\{(F, H): F \in R_{*}^{N \backslash P_{l}}, H \in R^{P_{l}}, i \in N_{H},\left|M^{r}(F)\right|=\left|M^{r}(L)\right| \text { and } \forall k \in R,\left|H_{k}\right|=\left|G_{k}\right|\right\} \\
& =\left\{(F, H) ; F \in R_{*}^{N \backslash P_{l}}, H \in R^{P_{l}}, i \in N_{H}, M^{r}(F) \in[L]_{l} \text { and } \forall k \in R,\left|H_{k}\right|=\left|G_{k}\right|\right\} \\
& =\left\{(F, H) ; F \in R_{*}^{N \backslash P_{l}}, H \in R^{P_{l}}, i \in \cup_{j \in R} H_{j}, M^{r}(F) \in[L]_{l} \text { and } \forall k \in R,\left|H_{k}\right|=\left|G_{k}\right|\right\} \\
& =\bigcup_{j \in R}\left\{(F, H) ; F \in R_{*}^{N \backslash P_{l}}, H \in R^{P_{l}}, i \in H_{j}, M^{r}(F) \in[L]_{l} \text { and } \forall k \in R,\left|H_{k}\right|=\left|G_{k}\right|\right\} \\
& =\bigcup_{j \in R} \bar{W}_{l}^{i, j}
\end{aligned}
$$

where: $\bar{W}_{l}^{i, j}=\left\{(F, H): F \in R_{*}^{N \backslash P_{l}}, H \in R^{P_{l}}, i \in H_{j}, M^{r}(F) \in[L]_{l}\right.$ and $\left.\forall k \in R,\left|H_{k}\right|=\left|G_{k}\right|\right\}$

$$
\begin{aligned}
& =\left\{F \in R_{*}^{N \backslash P_{l}}: M^{r}(F) \in[L]_{l}\right\} \times\left\{H \in R^{P_{l}}, i \in H_{j}, \forall k \in R,\left|H_{k}\right|=\left|G_{k}\right|\right\} \\
& =\left(\bigcup_{I \in[L]_{l}}\left\{F \in R_{*}^{N \backslash P_{l}}: M^{r}(F)=I\right\}\right) \times\left\{H \in R^{P_{l}}: i \in H_{j}, \forall k \in R,\left|H_{k}\right|=\left|G_{k}\right|\right\}
\end{aligned}
$$

Moreover, $\Phi_{i}(V, P)=\sum_{F \in R_{*}^{N \backslash P_{l}}}\left(\sum_{H \in R^{P_{l}}} f_{l}(F, H)[V(F \cup H)-\bar{V}(F \cup(H-i))]\right)$. 
For all $(F, H) \in \bar{W}_{l}^{i}$, we have $\left|M^{r}(F)\right|=\left|M^{r}(L)\right|, \forall k \in R,\left|H_{k}\right|=\left|G_{k}\right|$ and $f_{l}(F, H)=$ $f_{l}(L, G)$ holds thanks to the Equal Size axiom.

$$
\begin{aligned}
& \text { Thus, } \Phi_{i}(V, P)=\sum_{(L, G) \in \bar{W}_{l}^{i}} f_{l}(L, G)=f_{l}(L, G)\left|\bar{W}_{l}^{i}\right|=f_{l}(L, G)\left|\cup \bar{W}_{j \in R}^{i, j}\right|=f_{l}(L, G) \sum_{j \in R}\left|\bar{W}_{l}^{i, j}\right| \\
= & f_{l}(L, G) \sum_{j \in R}\left|\left[\bigcup_{I \in[L]_{l}}\left\{F \in R_{*}^{N \backslash P_{l}}: M^{r}(F)=I\right\}\right] \times\left\{H \in R^{P_{l}}: i \in H_{j}, \forall k \in R,\left|H_{k}\right|=\left|G_{k}\right|\right\}\right| \\
= & f_{l}(L, G)\left[\sum_{I \in[L]_{l}}\left|\left\{F \in R^{N \backslash P_{h}}: M^{r}(F)=I\right\}\right|\right]\left[\sum_{j \in R}\left|\left\{H \in R^{P_{l}}, i \in H_{j}: \forall k \in R,\left|H_{k}\right|=\left|G_{k}\right|\right\}\right|\right]
\end{aligned}
$$

However, $\forall I \in[L]_{l},\left|\left\{F \in R_{*}^{N \backslash P_{l}}: M^{r}(F)=I\right\}\right|=r^{\left(\sum_{h \in I}\left|P_{h}\right|\right)}$ and thanks to Lemma 2, we have: for all $j \in R,\left|\left\{H \in R^{P_{l}}, i \in H_{j}: \forall k \in R,\left|H_{k}\right|=\left|G_{k}\right|\right\}\right|=\frac{\left|P_{l}-1\right| !}{\left(\left|P_{l}\right|-\sum_{k \in R}\left|G_{k}\right|\right) !\left(\prod_{k \in R, k \neq j}\left|G_{k}\right| !\right)\left(\left|G_{j}\right|-1\right) !}$

$$
=\frac{\left|P_{l}-1\right| !\left|G_{j}\right|}{\left(\left|P_{l}\right|-\sum_{k \in R}\left|G_{k}\right|\right):\left(\prod_{k \in R}\left|G_{k}\right| !\right)} .
$$

Thus, $\Phi_{i}(V, P)=f_{l}(L, G)\left(\sum_{I \in[L]_{l}} r_{\left(\sum_{h \in I}\left|P_{h}\right|\right)}\right)\left(\frac{\left|P_{l}-1\right| ! \sum_{j \in R}\left|G_{j}\right|}{\left(\left|P_{l}\right|-\sum_{k \in R}\left|G_{k}\right|\right) !\left(\prod_{k \in R}\left|G_{k}\right| !\right)}\right)$ and finally, by Equal Share, $\forall i \in P_{l}, \Phi_{i}(V, P)=\frac{1}{m\left|P_{l}\right|}$, that is $f_{l}(L, G)\left(\sum_{I \in[L]_{l}} r_{l}\left(\sum_{h \in I}\left|P_{h}\right|\right)\right)\left(\frac{\left|P_{l}-1\right| ! \sum_{j \in R}\left|G_{j}\right|}{\left(\left|P_{l}\right|-\sum_{k \in R}\left|G_{k}\right|\right) !\left(\prod_{k \in R}\left|G_{k}\right| !\right)}\right)=\frac{1}{m\left|P_{l}\right|}$, which implies that:

$$
\begin{aligned}
f_{l}(L, G) & =\frac{1}{m\left|P_{l}\right|} \times\left[\sum_{I \in[L]_{l}} r\left(\sum_{h \in I}\left|P_{h}\right|\right)\right]^{-1}\left[\frac{\left(\left|P_{l}\right|-\sum_{k \in R}\left|G_{k}\right|\right) !\left(\prod_{k \in R}\left|G_{k}\right| !\right)}{\left|P_{l}-1\right| ! \sum_{j \in R}\left|G_{j}\right|}\right] \\
& =\left[m \sum_{I \in[L]_{l}} r\left(\sum_{h \in I}\left|P_{h}\right|\right)\right]^{-1}\left[\frac{\left(\left|P_{l}\right|-\sum_{k \in R}\left|G_{k}\right|\right) !\left(\prod_{k \in R}\left|G_{k}\right| !\right)}{\left|P_{l}\right| !\left(\sum_{k \in R}\left|G_{k}\right|\right)}\right]
\end{aligned}
$$


$\Leftarrow)$ Conversely, let $\Phi$ be a CSWD power index such that

$$
f_{l}(L, G)=\left[m \sum_{I \in[L]_{l}} r\left(\sum_{h \in I}\left|P_{h}\right|\right)\right]^{-1}\left[\frac{\left(\left|P_{l}\right|-\sum_{k \in R}\left|G_{k}\right|\right) \mid\left(\prod_{k \in R}\left|G_{k}\right| !\right)}{\left|P_{l}\right| !\left(\sum_{k \in R}\left|G_{k}\right|\right)}\right]
$$

- It is obvious that $\Phi$ satisfies the Equal Size condition.

- Let us now prove that $\Phi$ satisfies the Equal share axiom.

Consider a DMGCs which is $l$-anonymous with $\forall F \in R^{N}, V(F)=1$ if and only if $\left[\left|M^{r}\left(F^{-l}\right)\right| \geq \mu_{l}\right]$ or $\left[\left|M^{r}\left(F^{-l}\right)\right|=\mu_{l}-1\right.$ and $\left.\forall k \in R,\left|F_{k} \cap P_{l}\right| \geq q_{k}^{l}\right]$.

We have: $\Phi_{i}(V, P)=\sum_{L \in R_{*}^{N \backslash P_{l}}}\left(\sum_{G \in R^{P_{l}}} f_{l}(L, G)[V(L \cup G)-\bar{V}(L \cup(G-i))]\right)=\sum_{(L, G) \in \bar{W}_{l}^{i}} f_{l}(L, G)$, where $\bar{W}_{l}^{i}=\left\{(L, G): L \in R_{*}^{N \backslash P_{l}}, G \in R^{P_{l}}\right.$ and $\left.V(L \cup G)-\bar{V}(L \cup(G-i))=1\right\}$. We know from Lemma 3 that if $(L, G) \in W_{l}^{i}$, then $\left|M^{r}(L)\right|=\mu_{l}-1$ and $\forall k \in R,\left|G_{k}\right|=q_{k}^{l}$, which implies that $f_{l}(L, G)$ does not depend on $(L, G)$ and is given by :

$$
f_{l}(L, G)=\left(m \sum_{I \in[L]_{l}} r_{h \in I}\left(\sum_{h \in I}\left|P_{h}\right|\right)\right)^{-1}\left(\frac{\left(\left|P_{l}\right|-\sum_{k \in R}\left|q_{k}^{l}\right|\right) \mid !\left(\prod_{k \in R}\left|q_{k}^{l}\right| !\right)}{\left|P_{l}\right| !\left(\sum_{k \in R}\left|q_{k}^{l}\right|\right)}\right)
$$

Hence, $\Phi_{i}(V, P)=f_{l}(L, G)\left|\bar{W}_{l}^{i}\right|$. As we did in the first part of the proof, let us remark that $\bar{W}_{l}^{i}=\cup_{j \in R} X_{l}^{i, j}$, where

$$
\begin{aligned}
X_{l}^{i, j} & =\left\{(F, H) \in \bar{W}_{l}^{i}: i \in H_{j}\right\} \\
& =\left\{(F, H): F \in R_{*}^{N \backslash P_{l}}, H \in R^{P_{l}}, i \in G_{j}, M^{r}(F)=\mu_{l}-1 \text { and } \forall k \in R,\left|H_{k}\right|=q_{k}^{l}\right\} \\
& =\left\{F \in R_{*}^{N \backslash P_{l}}: M^{r}(F)=\mu_{l}-1\right\} \times\left\{H \in R^{P_{l}}: i \in H_{j}, \forall k \in R,\left|H_{k}\right|=q_{k}^{l}\right\} \\
& =\left\{F \in R_{*}^{N \backslash P_{l}}: M^{r}(F)=M^{r}(L)\right\} \times\left\{H \in R^{P_{l}}: i \in H_{j}, \forall k \in R,\left|H_{k}\right|=q_{k}^{l}\right\} \\
& =\left\{F \in R_{*}^{N \backslash P_{l}}: F \in[L]\right\} \times\left\{H \in R^{P_{l}}: i \in H_{j}, \forall k \in R,\left|H_{k}\right|=q_{k}^{l}\right\} \\
& =\left\{\bigcup_{I \in[L]}\left\{F \in R_{*}^{N \backslash P_{l}}: M^{r}(F)=I\right\}\right) \times\left\{H \in R^{P_{l}}, i \in H_{j}: \forall k \in R,\left|H_{k}\right|=q_{k}^{l}\right\}
\end{aligned}
$$


Thus, $\left|\bar{W}_{l}^{i}\right|=\left|\cup X_{j \in R}^{i, j}\right|=\sum_{j \in R}\left|X_{l}^{i, j}\right|$

$$
\begin{aligned}
& =\sum_{j \in R}\left|\left(\bigcup_{I \in[L]_{l}}\left\{F \in R_{*}^{N \backslash P_{l}}: M^{r}(F)=I\right\}\right) \times\left\{G \in R^{P_{l}}: i \in G_{j}, \forall k \in R,\left|G_{k}\right|=q_{k}^{l}\right\}\right| \\
& =\left[\sum_{I \in[L]_{l}}\left|\left\{F \in R_{*}^{N \backslash P_{l}}: M^{r}(F)=I\right\}\right|\right]\left[\sum_{j \in R}\left|\left\{G \in R^{P_{l}}: i \in G_{j}, \forall k \in R,\left|G_{k}\right|=q_{k}^{l}\right\}\right|\right]
\end{aligned}
$$

For all $j \in R,\left|\left\{G \in R^{P_{l}}, i \in G_{j}: \forall k \in R,\left|G_{k}\right|=q_{k}^{l}\right\}\right|$ is the number of partitions of $N-i$ into $r+1$ coalitions $G_{1}, G_{2}, \ldots, G_{r}$ and $G_{r+1}$ such that $\left|G_{1}\right|=q_{1}^{l}, \ldots,\left|G_{j-1}\right|=q_{j-1}^{l}$, $\left|G_{j}\right|=q_{j}^{l}-1$, (by assuming that $\left.i \in G_{j}\right),\left|G_{j+1}\right|=q_{j+1}^{l}, \ldots,\left|G_{r}\right|=q_{r}$ and $\left|G_{r+1}\right|=(n-$ $\left.\sum_{k \in R}\left|G_{k}\right|\right)$. As above, we obtain: $\left|\left\{G \in R^{P_{l}}, i \in G_{j}: \forall k \in R,\left|G_{k}\right|=q_{k}^{l}\right\}\right|=\frac{\left|P_{l}-1\right| !\left|q_{j}^{l}\right|}{\left(\left|P_{l}\right|-\sum_{k \in R}\left|q_{k}^{l}\right|\right) !\left(\prod_{k \in R} q_{k}^{l} !\right.}$.

Further, for all $I \in[L]_{l},\left|\left\{F \in R_{*}^{N \backslash P_{l}}: M^{r}(F)=I\right\}\right|=r\left(\sum_{h \in I}\left|P_{h}\right|\right)$.

Hence, $\left|\bar{W}_{l}^{i}\right|=\left(\sum_{I \in[L]_{l}} r\left(\sum_{h \in I}^{\left.\left|P_{h}\right|\right)}\right)\left(\sum_{j \in R} \frac{\left|P_{l}-1\right| !\left|q_{j}^{l}\right|}{\left(\left|P_{l}\right|-\sum_{k \in R}\left|q_{k}^{l}\right|\right) !\left(\prod_{k \in R} q_{k}^{l} !\right.}\right)\right)$ and finally,

$$
\begin{aligned}
\Phi_{i}(V, P) & =f_{l}(L, G)\left|\bar{W}_{l}^{i}\right| \\
& =\left(m \sum_{I \in[L]_{l}} r\left(\sum_{h \in I}\left|P_{l}\right|\right)\right)^{-1}\left(\frac{\left(\left|P_{l}\right|-\sum_{k \in R}\left|q_{k}^{l}\right|\right) !\left(\prod_{k \in R}\left|q_{k}^{l}\right| !\right)}{\left|P_{l}\right| !\left(\sum_{k \in R}\left|q_{k}^{l}\right|\right)}\right) \times\left(\sum_{I \in[L]_{l}} r\left(\sum_{h \in I}\left|P_{h}\right|\right)\right)\left(\sum_{j \in R} \frac{\left|P_{l}-1\right| !\left|q_{j}\right|}{\left(\left|P_{l}\right|-\sum_{k \in R}\left|q_{k}^{l}\right|\right) ! \mid\left(\prod_{k \in R} q_{k}^{l} !\right)}\right) \\
& =\frac{1}{m\left|P_{l}\right|}
\end{aligned}
$$

This concludes the proof. 


\section{A.3 Proof of corollary 1}

Proof. Let $|R|=1, l \in M, L \in R_{*}^{N / P_{l}}$ and $G \in R^{P_{l}}$, then there exist $T \in 2_{*}^{N \backslash P_{l}}$ and $S \subseteq P_{l}$ such that: $L=\{T\}$ and $G=\{S\}$. We have,

$$
\begin{aligned}
f_{l}(L, G)=f_{l}(T, S) & \left.=\left[m \sum_{I \in[L]_{l}} 1 \sum_{h \in I}\left|P_{h}\right|\right)\right]^{-1}\left[\frac{\left(\left|P_{l}\right|-\sum_{k \in\{1\}}\left|G_{k}\right|\right) !\left(\prod_{k \in\{1\}}\left|G_{k}\right| !\right)}{\left|P_{l}\right| !\left(\sum_{k \in\{1\}}\left|G_{k}\right|\right)}\right] \\
& =\left[m \sum_{I \in[L]_{l}} 1\right]^{-1}\left[\frac{\left(\left|P_{l}\right|-|S|\right) !|S| !}{\left|P_{l}\right| !|S|}\right] \\
& =[m|\{I \subseteq M-l:|I|=|M(L)|\}|]^{-1}\left[\frac{\left(\left|P_{l}\right|-|S|\right) !(|S|-1) !}{\left|P_{l}\right| !}\right] \\
& =\left[m C_{m-1}^{|M(T)|}\right]^{-1}\left[\frac{\left(\left|P_{l}\right|-|S|\right) !(|S|-1) !}{\left|P_{l}\right| !}\right], \text { note that }|M(L)|=|M(T)| \\
& =\frac{\mid M(T) ! !(m-|M(T)|-1) !}{m !} \frac{(|S|-1) !\left(\left|P_{\mid}\right|-|S|\right) !}{\left|P_{P}\right| !}
\end{aligned}
$$

It follows that: $\Phi_{i}(V, P)=\sum_{L \in R_{*}^{N \backslash P_{l}}} \sum_{G \in R^{P_{l}}} f_{l}(L, G)[V(L \cup G)-\bar{V}(L \cup(G-i))]$

$$
\begin{aligned}
& =\sum_{T \in 2_{*}^{N \backslash P} l} \sum_{S \subseteq P_{l}, i \in S} f_{l}(T, S)[V(S \cup T)-V((S-i) \cup T)] \\
& =\sum_{T \in 2_{*}^{N \backslash P_{l}}} \sum_{S \subseteq P_{l}, i \in S} \frac{\mid M(T) !(m-|M(T)|-1) !}{m !} \frac{(|S|-1) ! !\left(P_{P}|-| S \mid\right) !}{\left|P_{l}\right| !}[V(S \cup T)-V((S-i) \cup T)] \\
& =O S_{i}(V, P)
\end{aligned}
$$

This concludes the proof.

\section{A.4 Proof of proposition 1}

Proof. $\Rightarrow)$ Let $\varphi_{i}(v, P)$ be a CSWD. Assume further that $\varphi$ is $\left(\lambda^{l}\right)$-constant-weight. Then, for all $T \in 2_{*}^{N \backslash P_{l}}$ and for all $S \subseteq P_{l}, f_{l}(T, S)=\lambda^{l}$. Let $(v, P)$ a be $S G C s$ and $i \in P_{l}$, 
we have:

$$
\begin{aligned}
\varphi_{i}(v, P) & =\sum_{T \in 2_{*}^{N \backslash P_{l}}} \sum_{S \subseteq P_{l}, i \in S} f_{l}(T, S)[v(S \cup T)-v((S-i) \cup T)] \\
& =\sum_{T \in 2_{*}^{N} \backslash P_{l}} \sum_{S \subseteq P_{l}, i \in S} \lambda^{l}[v(S \cup T)-v((S-i) \cup T)] \\
& =\lambda^{l} \sum_{T \in 2_{*}^{N} P_{l} S \subseteq P_{l}, i \in S}[v(S \cup T)-v((S-i) \cup T)] \\
& =\lambda^{l} \eta_{i}(v, P)
\end{aligned}
$$

$\Leftarrow)$ The converse is straightforward.

\section{A.5 Proof of Proposition 2}

Proof. Let $\Phi$ be a CSWD power index. If $\Phi$ is $\left(\delta^{l}\right)$-constant-weight, then for all $i \in N$,

$$
\begin{aligned}
& \Phi_{i}(V, P)=\sum_{L \in R_{*}^{N \backslash P_{l}}} \sum_{G \in R^{P_{l}}} f_{l}(L, G)[V(L \cup G)-\bar{V}(L \cup(G-i))] \\
& =\sum_{L \in R_{*}^{N \backslash P_{l}}} \sum_{G \in R^{P_{l}}} \delta^{l}[V(L \cup G)-\bar{V}(L \cup(G-i))]
\end{aligned}
$$

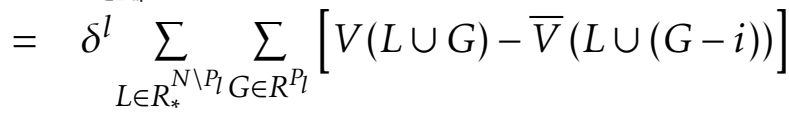

$$
\begin{aligned}
& =\delta^{l} \eta_{i}^{r}(V, P) \text {. }
\end{aligned}
$$

$\Leftarrow)$ The converse is straightforward. 